\title{
Distributed Heterogeneous Outdoor Multi-robot Localization ${ }^{\dagger}$
}

\author{
Raj Madhavan, Kingsley Fregene ${ }^{\ddagger}$ and Lynne E. Parker \\ Center for Engineering Science Advanced Research \\ Computer Science and Mathematics Division \\ Oak Ridge National Laboratory \\ Oak Ridge, TN 37831-6355, U.S.A. \\ E-mail: raj_madhavan@yahoo.com, kocfrege@ieee.org, parkerle@ornl.gov \\ Fax: (865) 241-0381.
}

\begin{abstract}
An Extended Kalman Filter-based algorithm for the localization of a team of robots is described in this paper. The distributed EKF localization scheme is straightforward in that the individual robots maintain a pose estimate using EKFs that are local to every robot. We then show how these results can be extended to perform heterogeneous cooperative localization in the absence or degradation of absolute sensors aboard the team members. The proposed algorithms are implemented using field data obtained from a team of ATRV-Mini robots traversing on uneven outdoor terrain.
\end{abstract}

Keywords - Multi-robot Localization, Heterogeneous Sensing, Distributed State Estimation, Cooperative Navigation.

\section{Introduction}

$\mathrm{T}$ HE development of unmanned mobile robotic systems that operate in complex and highly dynamic environments has received tremendous interest from roboticists in recent years. In the last decade or so, the notion of having a team of robots cooperating to achieve a goal has gained due attention since there are several advantages in having a group of robots cooperate to complete a required task in various application domains [1],[2]. Understandably, the reliability of such a system is much higher than single-robot systems, enabling the team to accomplish the intended mission goals even if one member of the team fails. However, the realization of a multi-robot system is not without difficulties and involves a much higher order of complexity when compared to single-robot systems.

Two types of architectures, centralized and decentralized, can be employed to achieve cooperation in a robot team. In a centralized architecture, all planning, execution control and monitoring tasks are performed by a single control unit. It is considerably difficult to

\footnotetext{
${ }^{\dagger}$ The research reported in this paper has been authored by contractors of the U.S. Government under Contract No. DE-AC0500OR22725 with UT-Battelle, LLC. The U.S. Government retains a nonexclusive royalty-free license to publish or reproduce the published form of this contribution, or allow others to do so, for U.S. Government purposes. Research sponsored by the Engineering Research Program of the Office of Basic Energy Sciences, U.S. Department of Energy.

$¥$ Currently with the Department of Electrical and Computer Engineering, University of Waterloo, Canada.
}

have a fully centralized scheme for the control of multiple robots as the increase in computational overhead is proportional to the number of robots in the team. When there is no central processing facility and all the decisional issues for a team member are tackled within the robot itself, the disadvantages associated with the centralized architectures can be overcome. This motivation has manifest itself into decentralized (and distributed) architectures that render robust and modular capabilities [3].

Localization, the process of determining the position and orientation (pose) of a robot within the operating environment, is critical for subsequent high level navigation tasks. Each member of the robot team considered in this paper possesses absolute positioning capability by means of differential GPS (DGPS). Multipathing ${ }^{1}$ errors make it extremely difficult to localize based on DGPS alone. Thus it becomes necessary to develop a framework within which observations from dead-reckoning and absolute sensors can be fused to continually deliver reliable and consistent pose estimates. Additionally, in order to minimize the computational bottlenecks associated with centralized architectures for multi-robot localization, we seek to decentralize the estimation process across the members of the group. To achieve the above requirements, a distributed Extended Kalman Filter-based algorithm for the localization of a team of robots operating on outdoor terrain is developed in this paper. In the first part of the paper, we show that distributed localization of robot team members can be realized in a straightforward manner by enabling each robot to maintain its pose using an EKF that is local to that particular robot. The resulting pose estimates are intended to aid the construction of an elevation map (described in a companion paper [4]). We also consider the case when

\footnotetext{
${ }^{1}$ Multipathing refers to the situation where the signals detected by the DGPS receiver have been reflected off surfaces prior to detection instead of following the straight line path between the satellite and the receiver.
} 
all of the robots may not possess absolute positioning capabilities either due to the absence of requisite sensors or due to the degradation of available sensors. We show in such cases how multi-robot cooperative localization can be achieved by exploiting heterogeneous sensors aboard the team members.

This paper is organized as follows: Section II briefly reviews previous cooperative navigation methodologies reported in the literature. Section III describes the distributed localization scheme. Section IV develops heterogeneous cooperative localization approaches. Section $\mathrm{V}$ provides the conclusions and indicates avenues by which the research proposed in this paper can be extended.

\section{Related Work}

Several robot-based cooperative navigation approaches have been presented in the literature $[5],[6],[7]$. The underlying idea in such schemes is that one member of the team uses another member of the team for realizing cooperative navigation. The main limitations of such approaches are that: 1) only one robot is allowed to move prior to the movement of the other members of the team, 2) the robots have to move in such a way that they can "see" each other, which necessitates visual (sensorial) contact to be maintained at all times, and 3) the coordination abilities of the team as a whole suffer as the tasks are carried out in groups.

Premvuti et. al describe a relative position localization system for multiple mobile robots [5]. All the robots in the team are equipped with a set of rotating laser beacons and a circular sensor array of photodiodes. The laser beam is projected onto the sensor array of the robots in the vicinity and the signals detected by the photodiodes are said to form a swept laser projection matrix pattern. The range of the neighboring robot is determined by the slope of the pattern and the location of the photodiode at the center of the pattern provides the direction. Another example of a robotbased strategy is the work by Bison et. al [6] where one robot tracks another robot carrying a beacon that produces a helicoidal light pattern with a camera and compares it to an a priori geometrical model to extract necessary information for pose estimation. Kurazume et. al [7] propose a cooperative positioning system where the cooperating robots are divided into two groups. One of the groups remains stationary and acts as a landmark while the other group moves. Then the landmark roles and movement between the two groups alternate until the goal position is reached. While there are some advantages to such a method (elimination of a priori knowledge of landmark locations, ability to determine three-dimensional positions), the aforementioned disadvantages remain.

Collaborative multi-robot localization, within an estimation-theoretic framework, have been considered by many authors. Fox et. al perform collaborative localization of two indoor robots equipped with a model of the environment using a sample-based version of the Monte Carlo Localization (MCL) algorithm [8]. The main strength of MCL is its ability to perform global localization. It is not clear how well it will perform when extended to unstructured domains and slippery terrain. Sanderson formulates the cooperative navigation problem in a Kalman filter (KF) framework [9]. In the proposed Cooperative Navigation System (CNS), inter-robot positions are treated as observations and the KF estimates the position of all robots simultaneously. The CNS algorithm has been tested on two Yamabico robots in an indoor environment equipped with ultrasonic sensors by which one robot can sense the position of the other robot. It is not clear whether a centralized or a decentralized methodology is adapted for exchange of sensing information and subsequent state estimate updates. The shortcomings of this formulation are: 1) each robot is assumed to move in prescribed trajectories, 2) the robots are assumed to be holonomic, and 3) the models describing robot motion are assumed to be linear.

Roumeliotis et. al present a KF-based distributed localization approach for cooperative localization [10]. A centralized KF performs data fusion by using observations from a group of mobile robots. Both proprioceptive (relative) and exteroceptive (absolute) sensors are employed. The standard KF prediction equations are decentralized and distributed among the robots in the team. It is argued that the multi-robot localization problem renders the state propagation equations of the centralized system to be decoupled with state coupling occurring only when relative pose observations become available. Whenever two robots meet, the relative pose information obtained from a camera tracking system is used to centrally update the pose estimates of the robot team members. The results are verified on an indoor robot team.

From the brief review above, it is evident that current cooperative robotic navigation research primarily concentrates on indoor environments. In outdoor environments, errors introduced due to distance traveled can be significant and unpredictable. This is a direct consequence of the undulatory nature of the terrain of travel and the uncertainties introduced into sensor data. These challenges make it comparatively difficult to realize successful navigation in unstructured outdoor environments. Motivated by these factors, this paper develops an EKF-based multi-robot heterogeneous localization framework similar to that developed by Roumeliotis et. al [10] but it differs from that approach in the following ways: 1) the kinematic model of the robots is nonlinear: A model that sufficiently 
captures the vehicle motion is key to efficient use of sensor data and is central to successful autonomous navigation. A nonholonomic robot with a nonlinear kinematic model performs significantly better as the model efficiently captures the maneuvers of the robot. 2) no absolute positioning system capable of providing relative pose information is assumed to be available: Even though we consider DGPS, it provides absolute position information for only a single robot (subject to the number of satellites in view at any given time), and 3) the robots traverse on uneven and unstructured outdoor terrain: Our approach would work better as we consider nonlinear kinematic models.

\section{Distributed EKF Localization}

The EKF employed for the localization of the robots requires a kinematic (process) model and a sensor (observation) model. The experimental setup, the process and observation models, the ensuing estimation cycle and the corresponding experimental results are presented in the following paragraphs.

\section{A. Experimental Setup and Sensor Calibration}

The experimental platform is a team of two ATRVMini wheeled mobile robots with 4 -wheel differentialdrive skid-steering. The experimental setup consists of a wireless mini-LAN, a Local Area DGPS (LADGPS), a software platform (mobility from RWI) and codes developed in-house under Linux to read and log the data for the sensors on each robot. The wireless LAN is setup outdoors between an Operator Console Unit (OCU) and the robots. The OCU consists of a rugged notebook equipped with a BreezeCOM access point and antennas. Each robot has a BreezeCOM station adapter and an antenna. The LADGPS is formed by the base station/antenna hardware connected to the OCU and remote stations/antennas directly mounted on each robot. Each robot's station receives differential corrections from the base station such that LADGPS accuracy of up to 10 centimeters is obtainable. The distributed CORBA-based interface offered by mobility ensures that querying the sensor slots of particular robots is done in a transparent decentralized manner by simply appending the robot's ID to all such queries.

The sensor suite for localization (shown in Figure 1) is comprised of encoders that measure the wheel speeds and heading, DGPS, compass, a pan-tilt-zoom (PTZ) capable camera for visual perception and a scanning laser rangefinder (not shown). Table I summarizes the sensor suite and its characteristics.

Since the experiments are carried out in an outdoor environment with the robots executing general motion (translation and rotation on all axes), sensor calibration is important to ensure accuracy of readings. For the encoder readings, external sensors (DGPS and magnetic compass) are used to obtain calibration factors corresponding to the various axes. The correction factor for magnetic compass is obtained by looking up geodesic charts to determine the angle of magnetic variation corresponding to the longitude/latitude of the experiment's location. During outdoor navigation, it is possible for the DGPS antenna to lose line of sight with the orbiting satellites (e.g., by going under a tree). To account for this, calibration trials were performed in which the error in the DGPS positions reported were obtained as a function of the number of satellites acquired.

\section{B. ATRV-Mini Kinematic Model}

In a 4-wheel differential-drive skid-steering configuration, the two wheels on the same side move in unison, with each pair on opposite sides capable of being driven independently. If both pairs are driven forward with the same speed, then the robot moves forward, but if they are driven in opposite directions, the robot will turn in place. For the ATRV-Mini, it is observed that the wheel pairs on the same side may also be rotated at different speeds thereby enabling the robot to make gradual turns as it traverses. This flexibility allows compact maneuvers to be effected, such as turning in place (i.e., executing a zero-radius turn). Skid-steering is difficult to model [11] as a skid-steering robot slips when it turns. It is our conclusion from the field trials that a no-slip kinematic model using encoder-based odometry in combination with external corrections from an absolute sensor suffices for localization.

The nominal (noise-free) discrete process model equations at discrete time-instants $k$ can be written as:

$$
\left[\begin{array}{l}
x_{v_{k}} \\
y_{v_{k}} \\
\phi_{v_{k}}
\end{array}\right]=\left[\begin{array}{l}
x_{v_{k-1}} \\
y_{v_{k-1}} \\
\phi_{v_{k-1}}
\end{array}\right]+\Delta T\left[\begin{array}{l}
V_{k} \cos \phi_{v_{k-1}} \\
V_{k} \sin \phi_{v_{k-1}} \\
\omega_{k}
\end{array}\right]
$$

where $\Delta T$ is the synchronous sampling interval between states at discrete-time instants $(k-1)$ and $k$. The control signals applied to the vehicle are $\mathbf{u}_{k}=$ $\left[V_{k}, \omega_{k}\right]$ where $V_{k}$ is the linear velocity and $\omega_{k}$ is the heading rate of the robot at time-instant $k$. The errors due to the control signals $V$ and $\omega$ are modeled as simple additive noise sources, $\delta V$ and $\delta \omega$, about their respective means $\bar{V}$ and $\bar{\omega}$ as $V_{k}=\bar{V}_{k}+\delta V_{k}$ and $\omega_{k}=\bar{\omega}_{k}+\delta \omega_{k}$. The error source vector is defined as: $\delta \mathbf{w}_{k}=\left[\delta V_{k}, \delta \omega_{k}\right]^{T}$ and is a direct effect of the associated modeling errors and uncertainty in control. The source errors $\delta V$ and $\delta \omega$ are assumed to be zeromean, uncorrelated Gaussian sequences with constant variances $\sigma_{V}^{2}$ and $\sigma_{\omega}^{2}$, respectively. The variances were determined experimentally to reflect true noise variances. 


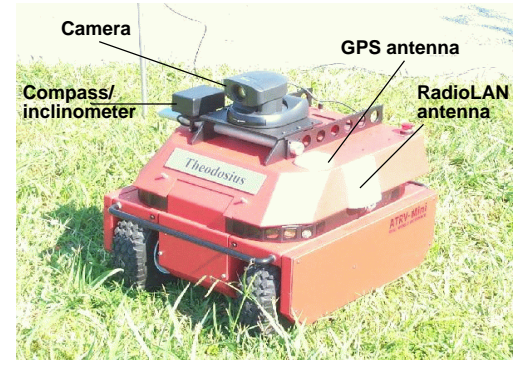

(a)

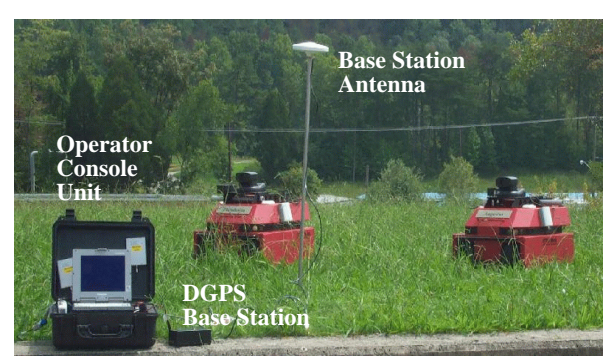

(b)

Fig. 1. The ATRV-Mini sensor suite and experimental setup. The sensor suite shown in (a) consists of encoders, DGPS, a compass and a PTZ camera (Also see Table I). The experimental setup depicted in (b) consists of an operator console unit, a DGPS base station and a base station antenna. See text for further details.

TABLE I

Sensor Suite Description

\begin{tabular}{||c|c|c|c||}
\hline Sensor & Type & Description & Freq. $(\mathbf{H z})$ \\
\hline \hline Odometry & relative & provides wheel speed and rate of change of heading & $\approx 50$ \\
\hline DGPS & absolute & provides $(x, y)$ position of robots & $\approx 1$ \\
\hline Vision & absolute & provides images using a pan-tilt-zoom $($ PTZ) capable camera & $\approx 10$ \\
\hline Compass & absolute & provides heading with respect to true north & $\approx 1$ \\
\hline Laser rangef inder & absolute & provides range and bearing to targets & $\approx 10$ \\
\hline
\end{tabular}

\section{Observation Models}

The observation model for DGPS and compass are given by:

$$
\begin{array}{ll}
\mathbf{z}_{k}^{\mathrm{g}}=\mathbf{H}_{k}^{\mathrm{g}} \mathbf{x}_{k}+v_{k}^{\mathrm{g}} ; & \mathbf{H}_{k}^{\mathrm{g}}=\left[\begin{array}{ccc}
1 & 0 & 0 \\
0 & 1 & 0
\end{array}\right] ; \\
z_{k}^{\mathrm{c}}=\mathbf{H}_{k}^{\mathrm{c}} \mathbf{x}_{k}+v_{k}^{\mathrm{c}} ; & \mathbf{H}_{k}^{\mathrm{c}}=\left[\begin{array}{lll}
0 & 0 & 1
\end{array}\right] ;
\end{array}
$$

where $v_{k}^{\mathrm{g}}$ and $v_{k}^{\mathrm{c}}$, respectively, refer to the uncertainty present in DGPS and compass observations, and are modeled as zero-mean uncorrelated Gaussian sequences with constant variances, $\left[\begin{array}{c}\sigma_{x_{g}}^{2} \\ \sigma_{y_{g}}^{2}\end{array}\right]$ and $\sigma_{\phi_{c}}^{2}$, respectively. The variances for the DGPS reported positions are set proportional to the number of satellites in view.

\section{Estimation Cycle}

The predict-observe-validate-update estimation cycle of the EKF for the localization of every robot team member proceeds as below:

- State Prediction: The state prediction takes place according to Equation (1) as follows:

$$
\begin{aligned}
& {\left[\begin{array}{l}
x_{v_{(k \mid k-1)}} \\
y_{v_{(k \mid k-1)}} \\
\phi_{v_{(k \mid k-1)}}
\end{array}\right]=\left[\begin{array}{l}
x_{v_{(k-1 \mid k-1)}} \\
y_{v_{(k-1 \mid k-1)}} \\
\phi_{v_{(k-1 \mid k-1)}}
\end{array}\right]} \\
& +\Delta T\left[\begin{array}{l}
V_{k} \cos \phi_{v_{(k-1 \mid k-1)}} \\
V_{k} \sin \phi_{v_{(k-1 \mid k-1)}} \\
\omega_{k}
\end{array}\right]
\end{aligned}
$$

The prediction covariance can now be computed using:

$$
\mathbf{P}_{(k \mid k-1)}=\nabla \mathbf{f}_{\mathbf{x}_{v_{k}}} \mathbf{P}_{(k-1 \mid k-1)} \nabla \mathbf{f}_{\mathbf{x}_{v_{k}}}^{T}+\nabla \mathbf{f}_{\mathbf{w}_{k}} \mathbf{Q}_{k} \nabla \mathbf{f}_{\mathbf{w}_{k}}^{T}
$$

where $\nabla \mathbf{f}_{\mathbf{x}_{v_{k}}}$ represents the Jacobian with respect to the states, $\nabla \mathbf{f}_{\mathbf{w}_{k}}$ is the Jacobian with respect to the error sources and $\mathbf{Q}_{k}$ is the noise strength matrix given by:

$$
\begin{aligned}
\nabla \mathbf{f}_{\mathbf{x}_{v_{k}}} & =\left[\begin{array}{lll}
1 & 0 & -\Delta T V_{k} \sin \phi_{v_{(k-1 \mid k-1)}} \\
0 & 1 & \Delta T V_{k} \cos \phi_{v_{(k-1 \mid k-1)}} \\
0 & 0 & 1
\end{array}\right] \\
\nabla \mathbf{f}_{\mathbf{w}_{k}} & =\Delta T\left[\begin{array}{ll}
\cos \phi_{v_{(k-1 \mid k-1)}} & 0 \\
\sin \phi_{v_{(k-1 \mid k-1)}} & 0 \\
0 & 1
\end{array}\right] \\
\mathbf{Q}_{\mathbf{k}} & =\left[\begin{array}{ll}
\sigma_{V_{k}}^{2} & 0 \\
0 & \sigma_{\omega_{k}}^{2}
\end{array}\right]
\end{aligned}
$$

- Observation Validation: Once the predicted states and their covariances are available, the DGPS/compass observations that arrive are accepted only if the observation falls inside the normalized residual validation gate, $\nu_{k}^{T} \mathbf{S}_{k}^{-1} \nu_{k} \leq \epsilon_{\gamma}$, where $\nu_{k}$ is the residual defined as the difference between the actual and predicted positions. The value of $\epsilon_{\gamma}$ can be chosen from the fact that the normalized residual sequence is a $\chi^{2}$ random variable with $m$ degrees of freedom ( $m$ being the dimension of the observation) [12]. The residual covariance is given by: $\mathbf{S}_{k}=\mathbf{H}_{k} \mathbf{P}_{(k \mid k-1)} \mathbf{H}_{k}^{T}+\mathbf{R}_{k}$. 
- Update: Once a validated observation is available, the state estimate and covariance updates are performed using the EKF update equations [12]:

$$
\begin{aligned}
\mathbf{x}_{(k \mid k)} & =\mathbf{x}_{(k \mid k-1)}+\mathbf{W}_{k} \nu_{k} \\
\mathbf{P}_{(k \mid k)} & =\mathbf{P}_{(k \mid k-1)}-\mathbf{W}_{k} \mathbf{S}_{k} \mathbf{W}_{k}^{T}
\end{aligned}
$$

where the Kalman gain matrix is given by

$$
\mathbf{W}_{k}=\mathbf{P}_{(k \mid k-1)} \mathbf{H}_{k}^{T} \mathbf{S}_{k}^{-1}
$$

\section{E. Experimental Results}

Figures 2(a)-(d) show the estimated path, orientation, pose standard deviations of the estimated pose and the $95 \%$ confidence $(2 \sigma)$ bounds for the DGPS residual, respectively, for one of the robots of the team. The EKF-based localization algorithm continually corrects the diverging dead-reckoning estimates based on external sensing information provided by DGPS and compass corrections as reflected by the periodic rise and fall of the pose standard deviations in Figure 2(c). The decrease in the standard deviations is due to the corrections offered by the DGPS/compass and the increase is due to the vehicle being localized based on prediction (dead-reckoning) alone. When the DGPS/compass do not provide aiding information towards the localization of the vehicle, the standard deviation is at a maximum. Due to the observation validation procedure, the residuals in (d) are clearly bounded and are indicative of consistent estimates.

\section{Outdoor Cooperative Localization Approaches}

When some robots of the team do not have absolute positioning capabilities or when the quality of the observations from the absolute positioning sensors deteriorate, another robot in the team (with better positioning capability) can assist in the localization of the robots whose sensors have deteriorated or failed. In such cases, if relative pose information is obtained, the EKF-based localization algorithm can be cast in a form such that the update stage of the EKF utilizes this relative pose thereby providing reliable pose estimates for all the members of the team. The observation model and two approaches for cooperative localization are detailed in the following paragraphs.

\section{A. Observation Model for Heterogeneous Cooperative Localization}

Let us consider the case when the team is composed of two robots. When robots \#1 and \#2 meet, they exchange relative pose information and the observation model becomes:

$$
\mathbf{z}_{c_{k}}=\left[\begin{array}{c}
x_{1_{k}}-x_{2_{k}} \\
y_{1_{k}}-y_{2_{k}} \\
\phi_{1_{k}}-\phi_{2_{k}}
\end{array}\right]+v_{12_{k}}=\mathbf{H}_{12_{k}} \mathbf{x}_{c_{k}}+v_{12_{k}}
$$

where $v_{12}$ refers to the uncertainty present in the relative pose observation and is modeled as a zero-mean uncorrelated Gaussian sequence with covariance $\mathbf{R}_{12_{k}}$.

The residual and the residual covariance are:

$$
\begin{aligned}
\nu_{c_{k}} & =\mathbf{z}_{c_{k}}-\hat{\mathbf{z}}_{c_{k}}=\mathbf{z}_{c_{k}}-\mathbf{H}_{12_{k}} \mathbf{x}_{c_{(k \mid k-1)}} \\
\mathbf{S}_{c_{k}} & =\mathbf{H}_{12_{k}} \mathbf{P}_{c_{(k \mid k-1)}} \mathbf{H}_{12_{k}}^{T}+\mathbf{R}_{12_{k}}
\end{aligned}
$$

The Kalman gain matrix, the state estimate and covariance updates (centralized) are as below:

$$
\begin{aligned}
& \mathbf{W}_{c_{k}}=\mathbf{P}_{c_{(k \mid k-1)}} \mathbf{H}_{12_{k}}^{T} \mathbf{S}_{c_{k}}^{-1} \\
& \mathbf{x}_{c_{(k \mid k)}}=\mathbf{x}_{c_{(k \mid k-1)}}+\mathbf{W}_{c_{k}}\left[\mathbf{z}_{c_{k}}-\left(\mathbf{x}_{1_{(k \mid k-1)}}-\mathbf{x}_{2_{(k \mid k-1)}}\right)\right] \\
& \mathbf{P}_{c_{(k \mid k)}}=\mathbf{P}_{c_{(k \mid k-1)}}-\mathbf{W}_{c_{k}} \mathbf{S}_{c_{k}} \mathbf{W}_{c_{k}}^{T} \\
& \text { where } \mathbf{x}_{c_{(k \mid k-1)}} \text { and } \mathbf{P}_{c_{(k \mid k-1)}} \text { are the state and covari- } \\
& \text { ance predictions, respectively. }
\end{aligned}
$$

\section{B. Laser-based Cooperative Localization}

Suppose that robot \#2 has a scanning laser rangefinder and also that the number of satellites in view from the current position of this robot indicates that DGPS is unavailable. (In the field trial, this corresponded to the robot going under a tree.) Given the pose of robot \#1, relative pose between robots \#2 and \#1 is determined as below:

1. Robot \#2 identifies robot \#1 and acquires a range and bearing laser scan.

2. After necessary preprocessing to discard readings that are greater than a predefined threshold, the range and bearing to the minima identified in the laser profile of robot \#1 are determined. An illustration of the identified minima marked by a square $(\square)$ is shown in Figure 3.

3. From the range and bearing pertaining to the minima, the pose of robot \#2 is inferred and relative pose information is now available for use in Equation (3).

Results: Within the EKF framework, state prediction takes place as before on individual robots according to Equation (2) in a decentralized and distributed fashion. By exchanging relative pose information, the states of the robots are updated in a centralized fashion as detailed in Section IV-A. The results for the laserbased cooperative localization are shown in Figure 4. Figure 4(a) shows the estimated paths of robots \#1 and $\# 2$. The pose standard deviations of robot\#2 in Figure 4(b) demonstrate the utility of the relative pose information in accomplishing cooperative localization. At time $=21$ seconds, DGPS becomes unavailable as indicated by the rise in the $x$ standard deviation. It can be seen that as a result of the laser-based relative position information, there is a sharp decrease in the position standard deviations of robot \#2 (marked by arrows). As the motion of the robot is primarily in the $x$ direction when the corrections are provided, the resulting decrease in the $x$ standard deviation is noticeable compared to those in $y$ and $\phi$. 


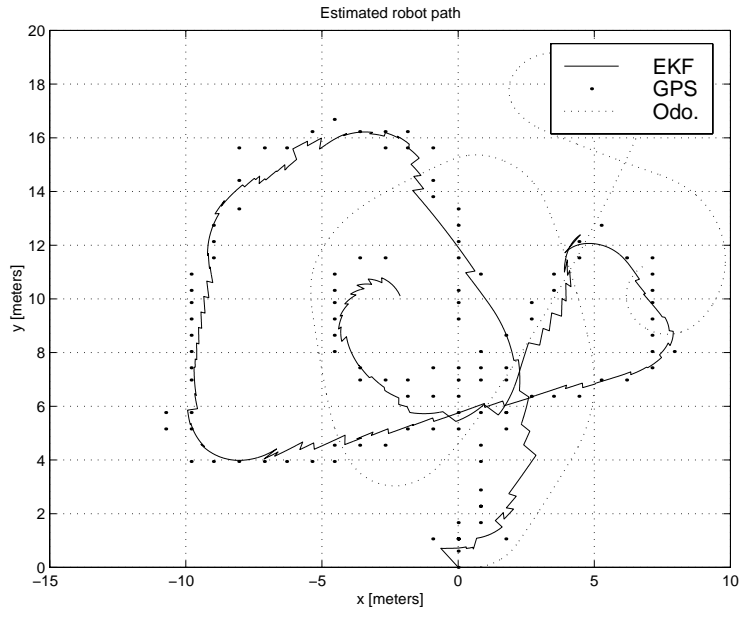

(a)
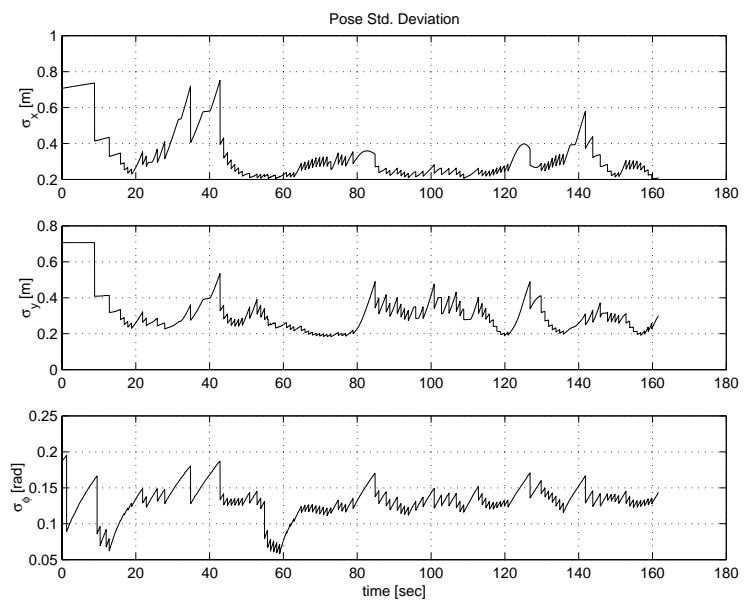

(c)

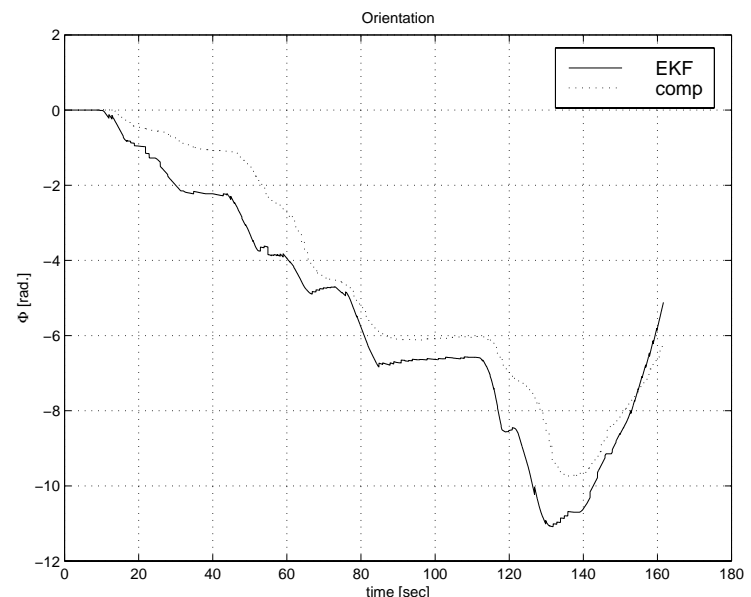

(b)
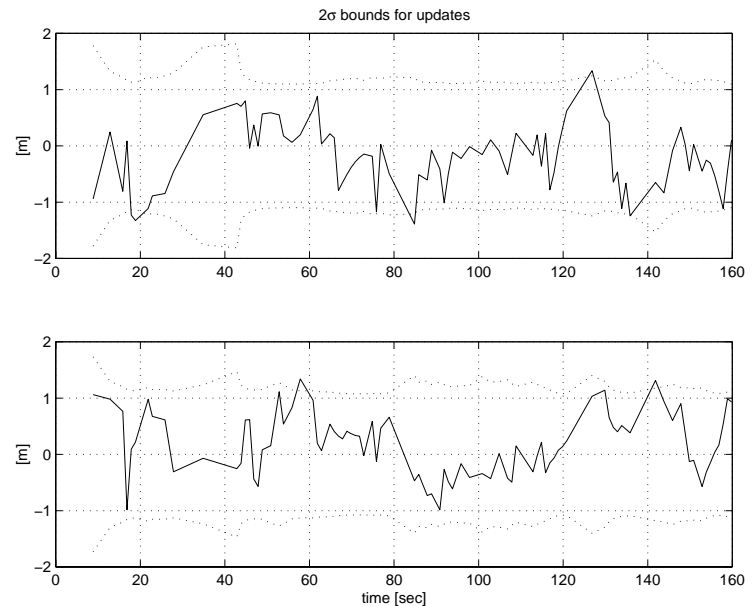

(d)

Fig. 2. EKF estimated robot path is shown in (a), orientation in (b), the standard deviation of the pose in (c) and the residual with the $95 \%(2 \sigma)$ confidence bounds in $(\mathrm{d})$. In $(\mathrm{a})$, the robot starts at $(0,0)$ and the dots represent the DGPS observations. The odometric path is plotted in (a) and the compass readings are plotted in (b) for comparison.

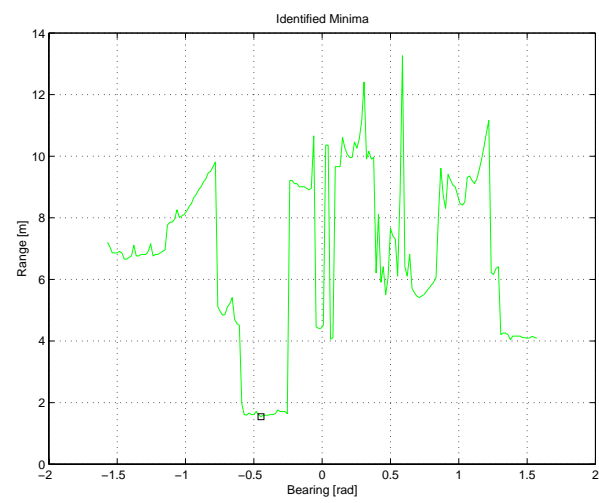

Fig. 3. The square $(\square)$ denotes the identified minima from a laser rangefinder scan.

\section{Vision-based Cooperative Localization}

In this approach, the PTZ camera that is part of the sensor suite is used to provide relative position information. Consider the case where two robots are performing cooperative localization with the cameraequipped robot \#1 lacking in absolute positioning capability. Relative position information is obtained as follows: First, robot \#1 searches the vicinity for another robot (say, robot \#2) whose pose is known (this is determined via TCP/IP message passing). Robot \#1 then visually acquires robot \#2 using an object recognition algorithm. The algorithm identifies the centroid of the robot within the image frame using a color segmentation scheme and marks its pixel co- 


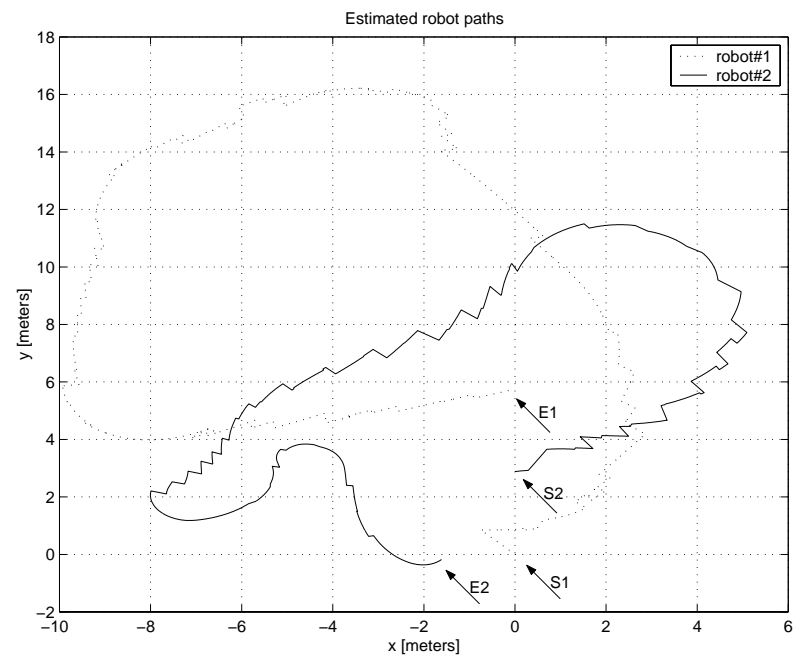

(a)
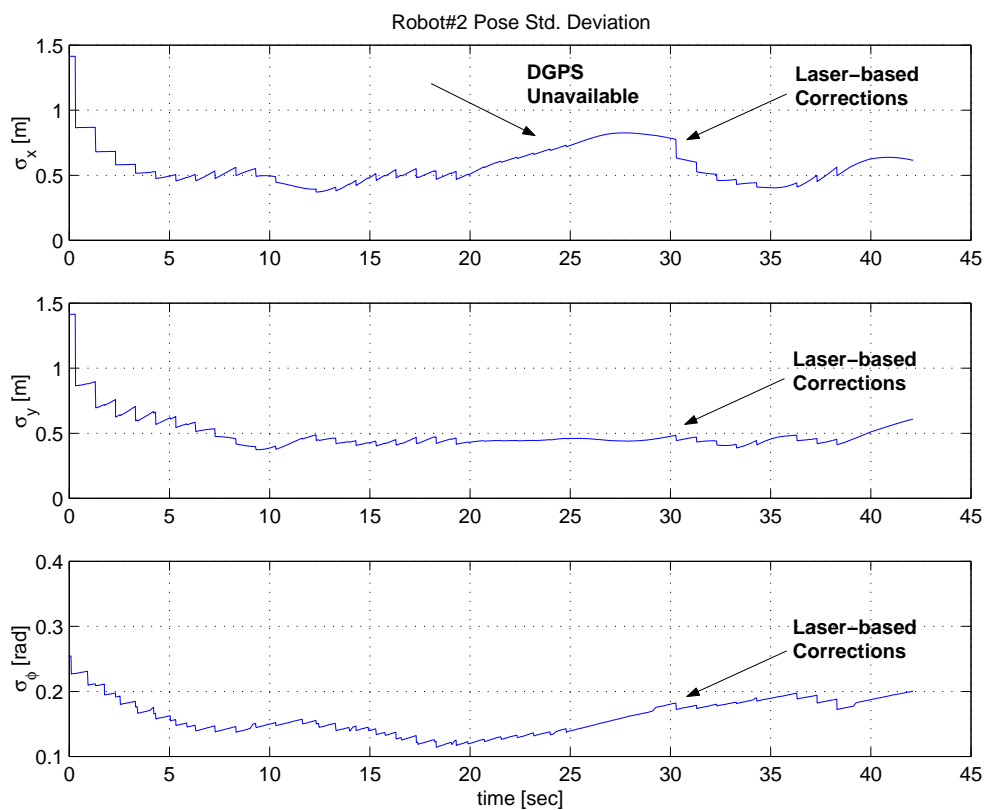

(b)

Fig. 4. Laser-based cooperative localization. The robots perform cooperative localization when DGPS becomes unavailable or when there are not enough satellites in view. EKF estimated robot paths are shown in (a). The solid line denotes the estimated path of robot \#2 and the dotted line that of robot \#1. The standard deviation of the pose of robot \#2 is shown in (b). The external corrections offered by the laser-based localization scheme are marked by arrows. (S1,E1) and (S2,E2) denote the start and end positions for robots \#1 and \#2, respectively.

ordinates on that frame. Figures 5(a)-(d) show the marked coordinates for various robot positions. An incremental depth-from-motion algorithm (see [4] for more details) computes the depth for a window within the frame which encloses these coordinates. Depth (range) $d_{k}$ is computed using [13]:

$$
\begin{aligned}
& {\left[\begin{array}{l}
\Delta x_{k} \\
\Delta y_{k}
\end{array}\right]=d_{k}\left[\begin{array}{ccc}
-f & 0 & x \\
0 & -f & y
\end{array}\right]\left[\begin{array}{l}
t_{x_{k}} \\
t_{y_{k}} \\
t_{z_{k}}
\end{array}\right]} \\
& +\left[\begin{array}{ccc}
\frac{x y}{f} & -\left(f+\frac{x^{2}}{f}\right) & y \\
f+\frac{y^{2}}{f} & -\frac{x y}{f} & -x
\end{array}\right]\left[\begin{array}{l}
r_{x_{k}} \\
r_{y_{k}} \\
r_{z_{k}}
\end{array}\right]
\end{aligned}
$$




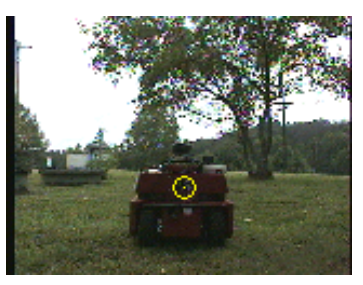

(a)

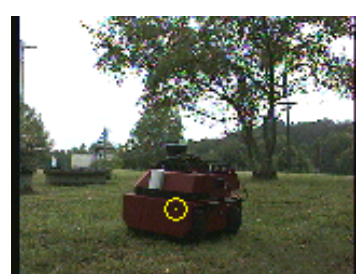

(b)

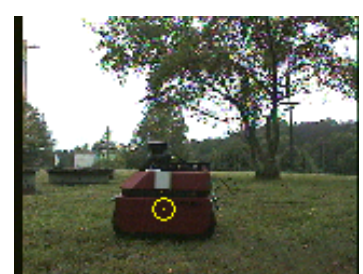

(c)

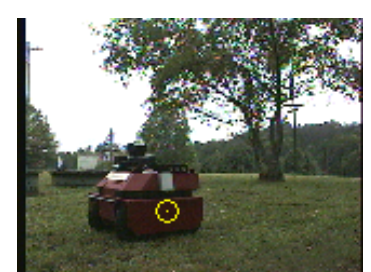

(d)

Fig. 5. Vision-based cooperative localization. The circles (o) denote the pixel coordinates (see text for details).

where $\left(\Delta x_{k}, \Delta y_{k}\right)$ is the optical flow between successive frames, $(x, y)$ are pixel coordinates, the triplets $\left(t_{x_{k}}, t_{y_{k}}, t_{z_{k}}\right)$ and $\left(r_{x_{k}}, r_{y_{k}}, r_{z_{k}}\right)$ are translational and rotational velocities, respectively, and $f$ is the camera focal length. The required relative position is inferred from the computed depth and the bearing of robot \#2 relative to robot \#1 is approximately determined from the lateral displacement between the enclosed pixel coordinates and the coordinates of the frame's optical center. The robot states are then updated as detailed in Section IV-A.

\section{Conclusions and Further Research}

A distributed EKF-based algorithm was described in this paper for the localization of a team of robots operating in uneven and unstructured environments. For cases where all robots of the team may not have absolute positioning capabilities, it was shown how cooperative localization can be performed exploiting heterogeneous sensors aboard the team members. The proposed scheme was successfully implemented using real data obtained from field trials with no modification to the operating environment nor to the robots of the team.

Further areas of research include the incorporation of slip within the kinematic model which will improve the prediction stage of the EKF. An entropy-based information metric developed in [14] can be used to evaluate the information content of an observation before that observation is used for cooperative localization thereby enabling the incorporation of observations that provide the maximum information towards localization. It is easy to utilize this metric within the proposed estimation-theoretic framework and such incorporation has already been proven to be effective in outdoor autonomous navigation [14]. Extended Information filtering (EIF) [12], a variant of EKF, has been widely touted in recent years as an antidote for the problems associated with EKF schemes. For the current application, the large matrix inversions necessitated by the EIF make it unsuitable. The extension of the information metric, the use of EIF and schemes for achieving global cooperative localization and their on- line implementation remain to be further investigated.

\section{Acknowledgment}

The authors would like to thank Mr. Hunter Brown of the North Carolina State University for his work on the object recognition algorithm described in Section IV-C.

\section{References}

[1] J. Feddama, C. Lewis, and R. Lafarge, "Cooperative Sentry Vehicles and Differential GPS Leapfrog," in Proceedings of Distributed Autonomous Robotic Systems, 2000, pp. 293302.

[2] D.W. Gage and J. Pletta, "Ground Vehicle Convoying," in Proceedings of the SPIE Conference on Mobile Robots II, Sept. 1987, vol. 852, pp. 319-327.

[3] L.E. Parker, "Current State of the Art in Distributed Robot Systems," in Proceedings of Distributed Autonomous Robotic Systems 4, 2000, pp. 3-12.

[4] K. Fregene, R. Madhavan, and L.E. Parker, "Incremental Multi-robot Terrain Mapping," Proceedings of the IEEE International Conference on Robotics and Automation (to appear), May 2002.

[5] S. Premvuti and J. Wang, "Relative Position Localizing System for Multiple Autonomous Robots in Distributed Robotic System: System Design and Simulation," Robotics and Autonomous Systems, vol. 18, pp. 319-326, 1996.

[6] P. Bison and G. Trainito, "A Robot Duo for Cooperative Autonomous Navigation," in Proceedings of Distributed Autonomous Robotic Systems 2, 1996, pp. 423-430.

[7] R. Kurazume, S. Hirose, S. Nagata, and N. Sashida, "Study on Cooperative Positioning System (Basic Principle and Measurement Experiment)," in Proceedings of the IEEE International Conference on Robotics and Automation, Apr. 1996, vol. 2, pp. 1421-1426.

[8] D. Fox, W. Burgard, H. Kruppa, and S. Thrun, "A Probabilistic Approach to Collaborative Multi-robot Localization," Autonomous Robots, vol. 8, no. 3, 2000.

[9] A.C. Sanderson, "Cooperative Navigation among Multiple Mobile Robots," in Proceedings of Distributed Autonomous Robotic Systems 2, 1996, pp. 389-400.

[10] S. Roumeliotis and G. Bekey, "Distributed Multi-Robot Localization," in Proceedings of Distributed Autonomous Robotic Systems. Oct. 2000, pp. 179-188, Springer-Verlag.

[11] J.T. Economou, Modelling and Control of Skid Steer Vehicles, Ph.D. thesis, Royal Military College of Science, Cranfield University, Sept. 1999.

[12] P. Maybeck, Stochastic Models, Estimation, and Control, vol. 1, Academic Press, New York, 1979.

[13] H.C. Lnguet-Higgins and K. Prazdny, "The Interpretation of a Moving Retinal Image," Proc. Roy. Soc. London, vol. B208, pp. 385-397, 1980.

[14] R. Madhavan, Terrain Aided Localisation of Autonomous Vehicles in Unstructured Environments, Ph.D. thesis, School of Aeronautical, Mechanical and Mechatronic Engineering, The University of Sydney, Jan. 2001. 